15 Tyrer P, Kendall T, Bateman A, Bayliss L, Bouras N, Burbeck R, et al. Draft NICE Guidelines for Consultation. Borderline Personality Disorder: Treatment and Management. NICE, 2008 (http://www.nice.org.uk/nicemedia/pdf/ BorderlinePersonalityDisorderFullGuidelineForConsultation.pdf)
16 Oldham JM, Bender DS, Skodol AE, Dyck IR, Sanislow CA, Yen S, et al. Testing an APA Practice Guideline: symptom-targeted medication utilization for patients with borderline personality disorder. Focus 2005; 3: 484-8.

\title{
COMMENTARY
}

\section{Treating personality disorder in community mental health teams}

\author{
Commentary on ... Use of psychotropic medication \\ among psychiatric out-patients with personality disorder ${ }^{\dagger}$ \\ Conor Duggan ${ }^{1}$
}

The Psychiatrist (2010), 34, 86-88, doi: 10.1192/pb.bp.109.026450

${ }^{1}$ University of Nottingham and Nottinghamshire Healthcare NHS Trust

Correspondence to Conor Duggan (Conor.Duggan@Nottingham.ac.uk)

\begin{abstract}
Summary Although personality disorders are prevalent conditions and the cause of considerable morbidity, their management remains controversial. The rationale for using psychotropic medication in these conditions is poorly articulated and its evidence base is weak. Nonetheless, psychiatrists continue to prescribe medication for this group as the accompanying paper makes clear. What is less clear is their reason for doing so, as there are frequent co-occurring conditions that might justify the use of medication in their own right. If the field is to progress, there is no substitute for well-designed randomised controlled trials and these ought to be a priority in the setting of any research agenda.
\end{abstract}

Declaration of interest None.
The recent National Institute for Health and Clinical Excellence (NICE) guidance on the role of medication, especially for borderline personality disorder, has provoked not only comment but concern among practitioners as the recommendations were unusually trenchant (i.e. medication should not be used routinely for this condition). ${ }^{1}$ What are clinicians to make of this guidance and how will it affect their practice? More generally, what is the practice in prescribing for personality disorder within community mental health teams (CMHTs)?

It is probably fair to say that we simply do not know the answer to this last question as few National Health Service (NHS) trusts systematically collect good-quality treatment data on their patients, and fewer still interrogate such data and publish it. Hence, the report from Baker-Glenn and her colleagues on the use of psychotropic medication for those with a primary diagnosis of personality disorder in a community mental health team is especially welcome. ${ }^{\dagger}$ The authors found that for the 107 patients considered, $77 \%$ had been prescribed at least one psychotropic medication. Not surprisingly, in almost half of the sample the most commonly prescribed medication was an antidepressant.

'See original paper, pp. 83-86, this issue.
The total annual cost across the 107 patients (6 were excluded) was almost $£ 37000$. An important point implied by the authors is that their sample comprised those who were actively seeking treatment (e.g. 56\% had a diagnosis of emotionally unstable personality disorder), whereas it is characteristic of many of those with personality disorder to reject treatment. ${ }^{2}$ Hence that 'iceberg' of unmet need among those with personality disorder who, despite significant morbidity, either never seek mental health interventions, or having sought them are summarily rejected, cannot be addressed by this survey - an omission candidly acknowledged by the authors.

Before making some general observations, it is worth commenting on one point which, to this reader at least, is unclear from the survey - and that is length of time during which the survey occurred. Although the authors state that this was based on the time 'during which they [the patients] had been under the care of the consultant', this, unfortunately, does not take us very much forward and it raises the following problem - the individual could have developed an Axis I condition in the past for which the medication might have been appropriately instituted. As comorbidity is the rule rather than the exception in those with personality disorder, ${ }^{3}$ with major depression being especially prevalent, it could have been the case that the prescribing of an 
antidepressant was entirely appropriate even if the primary disorder was personality disorder. Although the authors identify, and rightly exclude, six patients who had Axis I comorbidity, this comorbidity refers to serious and chronic disorders such as schizophrenia and bipolar affective disorder. No mention is made of the co-occurrence of other conditions such as depression that may have been the reason for at least initiating the medication. And this comorbidity of course represents real clinical experience where, as Claudius says to Gertrude in Hamlet, 'When sorrows come, they come not single spies, but in battalions.'

\section{Guidelines for medication in personality disorder}

Putting that uncertainty to one side, what can we say about medication for personality disorder in general that this survey and the recent NICE guidance highlight? First, there is a major problem with the nosology of personality disorders, as the current classification is recognised as being unhelpful in treatment selection - be this for either pharmacological or psychological interventions. ${ }^{4}$ Rather than treating individual personality disorders, the focus in the pharmacological treatment of personality disorders has been on behavioural clusters. Here, Paul Soloff ${ }^{5}$ has been influential, grouping symptom domains into: cognitive perceptual; affective (subdivided into disorders of (1) mood and (2) anxiety); impulse dyscontrol; and anxious fearful.

Broadly, for those with personality disorder, there is evidence for the use of conventional antipsychotic medication to reduce cognitive perceptual abnormalities and for selective serotonin reuptake inhibitors (SSRIs) to treat both affective dysregulation and impulsive dyscontrol. In addition, there is a range of anticonvulsants as well as mood stabilisers (including lithium and carbamazepine) that have some positive effects (for a review see Soloff ${ }^{5}$ ). As this list includes most of the psychiatric pharmacopeia and as individuals with personality disorder often present with combinations of symptoms either contemporaneously or sequentially, with many seeing these complex presentations as being the hallmark of personality disorder, these general recommendations are not seen as being especially helpful.

As mentioned above, personality disorder is often not only co-occurent with Axis I conditions, but is likely to exert a negative moderating influence on their outcome. ${ }^{6}$ The reasons for this are various (e.g. co-occurring excessive use of illicit drugs or alcohol, difficulties with adherence) but recognising this co-occurrence places an especially heavy obligation on the practitioner to combat these negative moderating influences.

Finally, it is worth commenting on the pressure on clinicians to 'do something' when faced with individuals in distress. This 'something must be done' pressure is difficult to withstand and no doubt leads to unwise prescribing that, as the authors observe, once commences may be difficult to stop. If the NICE guidance causes clinicians to think twice about their prescribing practices, then that ought to be a good thing.

Currently, we have personality disorder as a condition that is poorly defined and where the treatment recommendations are vague and often unhelpful. Although we have little evidence identifying drugs that might be effective, equally we have very little evidence that these same drugs are ineffective. This position of not knowing is an uncomfortable one for clinicians to be in so that it is understandable that they might respond in an extreme manner, veering in one direction or the other (i.e. overprescribing or underprescribing). Neither is especially helpful for the person in distress, but beyond Soloff's symptom clusters there is little to guide the practitioner at present.

\section{The way forward}

We certainly need more good quality clinical trials and one would think that the pharmacological industry would be keen to invest in identifying successful treatments for what is, after all, a chronic, if not a lifelong condition. That it has not done so is surprising. Moreover, it seems that there is now less interest in investigating what might be effective in this group compared with 10 years ago. Ideally, one would like head to head' trials (i.e. two active drugs being compared against one another) or a combination of drug and psychological intervention $v$. the drug alone, but given the relatively underdeveloped nature of the field, one will need to settle for examining the effectiveness of an active drug $v$. treatment as usual to at least commence this process of enquiry.

Underlying this reluctance to examine the efficacy of medication is perhaps the belief that personality disorder is largely a result of adverse early experience, so why should medication be effective? Although it is now firmly established that personality (and personality disorder) is the result of a complex interplay between a neurobiological substrate and psychosocial adversity, I believe that the simplistic dichotomous notion of psychological interventions for personality disorder and pharmacological interventions for Axis I conditions unfortunately still has a strong hold in the mind of practitioners in the field.

Hence, in this connection, I believe that our failure to take medication seriously may be a missed opportunity. Could it be, for instance, that this nihilism towards the efficacy of medication in personality disorder mirrors the attitude towards the use of chemotherapy in the treatment of cancer 30 years ago? Then, as indeed is the case currently with personality disorder, there was little evidence of the efficacy for any medication for the treatment of cancer, but this has changed radically over the intervening period. What has caused this change?

First, there was a commitment to large-scale, randomised controlled trials so that many (if not most) of those receiving interventions for cancer were enrolled in such trials as routine. This was remarkable given that those individuals were facing a life-threatening illness. The efficacy of new drugs in treating cancer has been identified by this process, but what has also been found to be efficacious is combining old drugs in new ways. It is possible therefore that we already have some of the answers for the effective pharmacological treatment for personality disorder under our noses, had we the will to discover it.

The NICE guideline rightly discourages the use of unrestrained polypharmacy for these disorders, yet I do have a concern that this might be taken too literally, so that psychiatrists in particular will take even less interest in what is already a marginalised group of service users. As an alternative to medication, the recommendation for borderline 
personality disorder is for well-resourced teams that should use well-integrated psychological interventions but that these should not be brief (i.e. of less than 3 months' duration). This is all well but these specialist teams will be costly to develop and maintain. Is there the stomach to fund these across the piece and is there capacity among the workforce to deliver what NICE recommends?

\section{Conclusions}

Not wishing to end on too negative a note, there is one professional who is suitably trained to manage complex cases and that is the general psychiatrist. Given that those with personality disorder will present not only with personality difficulties but with many other Axis I conditions that will be more resistant to conventional treatments because of the personality disorder, the full panoply of interventions - both pharmacological and psychological - will be required and properly sequenced. Is this not a tailor-made opportunity for psychiatrists to embrace under the New Ways of Working? Were this to occur, having a diagnosis of personality disorder would no longer be a reason for being excluded from services. This would be welcome and when it occurs, I suspect there will still be a place for medication, albeit on a more rational basis than is currently the case. Baker-Glenn et al's paper draws our attention to some of the many unanswered questions in this important area of psychiatric practice. It is high time for us to address these areas of ignorance!

\section{About the author}

Conor Duggan is Professor of Forensic Mental Health, University of Nottingham, and Honorary Consultant Psychiatrist, Nottinghamshire Healthcare NHS Trust

\section{References}

1 National Institute for Health and Clinical Excellence. Borderline Personality Disorder: Treatment and Management. The British Psychological Society and The Royal College of Psychiatrists, 2009.

2 Tyrer P, Mitchard S, Methuen C, Ranger M. Treatment-rejecting and treatment-seeking personality disorders: Type R and Type S. J Pers Disord 2003; 17: 265-70.

3 Swanson MC, Bland RC, Newman SC. Epidemiology of psychiatric disorders in Edmonton. Antisocial personality disorders. Acta Psychiatr Scand Suppl 1994; 376: 63-70.

4 Sanderson C, Clarkin JF. Further use of the NEO-Pi-R personality dimensions in differential treatment planning. In Personality Disorders and the Five Factor Model of Personality (2nd edn) (eds PT Costa, TA Widiger): 351-75. American Psychological Association Books, 2002.

5 Soloff PH. Symptom-orientated psychopharmacology for personality disorders. J Practical Psychiatr Behav Health 1998; 4: 3-11.

6 Reich JH, Green Al. Effect of personality disorders on outcome of treatment. J Nerv Ment Dis 1991; 192: 74-82.

\title{
Long-term follow-up of individuals on assertive outreach teams
}

\author{
Tanvir Rana, ${ }^{1}$ Martin Commander ${ }^{2}$
}

The Psychiatrist (2010), 34, 88-91, doi: 10.1192/pb.bp.108.022764

\begin{abstract}
${ }^{1}$ South Staffordshire and Shropshire Healthcare NHS Foundation Trust, Stafford; ${ }^{2}$ Birmingham and Solihull Mental Health Trust, Birmingham

Correspondence to Martin Commander (martin.commander@bsmhft.nhs.uk)
\end{abstract}

Aims and method To describe the long-term outcome of 165 people taken onto
assertive outreach teams. Results After a mean follow-up of 6 years and 8 months, 130 people remained in contact with local services of whom 100 were still under the care of an assertive outreach team. Admission rates remained around half those at inception. However, 16 individuals had died, 10 spent time in prison, 12 were homeless and 14 had protracted stays in hospital during the follow-up period.

Clinical implications Although assertive outreach teams are successful in engaging individuals and reducing admission rates, these benefits plateau after the first few years and could possibly be sustained by other services that may in addition focus more on those areas where teams have proven less effective including physical health, housing and employment.

Declaration of interest None.
The introduction of assertive outreach teams has been a central pillar of UK mental health policy in recent years. ${ }^{1}$ These teams focus on people with severe and enduring mental illness who have high levels of bed usage and are typically disaffected with services. Although they have been successful in engaging individuals and improving satisfaction, ${ }^{2}$ there is uncertainty about their ability to reduce demand on in-patient care, and findings with respect to 